\title{
MODELLING AND PARAMETERIZING PEDESTRIAN BEHAVIOUR IN PUBLIC PLACES: A REVIEW
}

\author{
Zsifkovits, M. \& Pham, T. S. \\ Institute for Theoretical Computer Science, Mathematics and Operations Research, \\ Universität der Bundeswehr Munich, 85577 Neubiberg, München, Germany \\ E-Mail: martin.zsifkovits@unibw.de,son.pham@unibw.de
}

\begin{abstract}
Literature and practice show a huge interest in modelling pedestrian behaviour, especially in public spaces. The most relevant applications are thereby traffic control, safety, and public security. Understanding the existing frameworks and finding possible measures and parameters for the models are a prerequisite to build a realistic model but very challenging. A literature review was conducted to collect, discuss and compare well-known and frequently cited models in order to derive implications for future models. The models were analysed from multiple points of view, namely the abstraction level, environment space, and behaviour types. Their parameterization was considered on both levels: microscopic and macroscopic. Advantages and disadvantages, difficulties and solution approaches of existing models were studied and discussed. Also, the data foundation is discussed, as a model should not only depend on artificial data and assumptions, which might lightly represent the subjective opinion of the model builder. Some technical approaches are proposed to overcome this difficulty. An analysis of various publications brings a structural and detailed perception about modelling pedestrian behaviour in crowds.

(Received in April 2017, accepted in September 2017. This paper was with the authors 2 months for 2 revisions.)
\end{abstract}

Keywords: Pedestrian Behaviour, Modelling Passenger Flow, Crowd Behaviour, Modelling Public Place, Evacuation

\section{INTRODUCTION}

The simulation of pedestrian behaviour has gained considerable interest in the last decades. The ability to simulate the behaviour and motion of crowds is not only cheaper but also less dangerous than real life experiments. Results are of interest to various institutions and companies, such as constructors of buildings, airplanes and ships; operators of large public constructions or mass events; or providers of public transport systems. Such system models allow for simulating evacuation scenarios in advance, discovering possible existing problems and optimizing plans [1]. Furthermore, the simulation of passengers' behaviour in public places compared to abnormal behaviour is of great interest for identifying possible terrorist threats.

Although several existing models have shown good results, there is no standard model for every situation. Building such models is therefore challenging each time, as there are no guidelines for setting the models' dimensions and parameters. By showing advantages and disadvantages of the existing models, this review offers an opportunity to learn from previous theory on a subject and understand it quickly. Additionally, difficulties in modelling pedestrian behaviour and their solution approaches will be shown and discussed. It is a precondition to build a realistic model or to improve an existing model fitting to a given situation.

Due to the increase in computational power over the past few decades, simulations of huge crowds are much more feasible today. However, the parameterization of such models is a challenging task, as behaviour and therefore data is extremely heterogeneous, difficult to access and it varies from sector to sector. It is therefore necessary to analyse existing empirical research to find possible measures and parameters for models. Additionally, it is 
decisive to pay attention to the model size for a long-term use, which is strongly related to the model complexity in most of time [2], and to understand correctly the contexts of existing models such as the characteristics of models and the modeller's skills [3]. A model becomes more complicated if the modeller is not the model user [3].

Literature shows that pedestrian behaviour adapts accordingly to the experienced situations. Zheng et al. [4] collected and divided typical phenomena into two situations: normal situations and emergencies. In normal situations, the typical phenomena are e.g. jamming, bi-direction movement, side-stepping, lane formation and free flow while in emergencies they are e.g. arching, clogging, following behaviour and faster-is-slower [4]. It is more difficult to model and simulate pedestrian behaviour in public than in private places. Since a pedestrian is used to a given situation, he reacts rather automatically and determined by his experience.

A brief overview on existing research will be given in the article at hand. Some wellknown and frequently cited models will be discussed from multiple points of view, namely abstraction level, environment space and behaviour types. Section 2 analyses existing empirical research, experiments and considers both the microscopic and the macroscopic level of description. In addition, it considers not only normal situations but also panic situations i.e. caused by fear in emergency situations or overcrowded places. Section 3 continues with the modelling approaches divided by the environment space, which are either space-discrete or space-continuous, to provide insights also in the modelling perspective. Section 4 considers the models according to behaviour type of pedestrians, which are either pre-programed rules based or learning techniques based as indicated by recent research. Finally, conclusions are given and the need for further research is discussed.

\section{ABSTRACTION LEVEL}

Although many people assume human behaviour to be very irregular and unpredictable, which may be true for extremely complex situations, it is possible to make some general rules for movement. According to the abstraction level, one might analyse this behaviour either on a microscopic, or on a macroscopic level.

\subsection{Microscopic level}

To react to their environment and sidestep obstacles, pedestrians observe their surroundings subconsciously [5]. This scanning area has the shape of "an ellipse which is narrow to either side of the individual and longest in front of him or her" [6]. The results of that scanning combined with the personal interests and aims of the individual are the stimulus which leads the person to physical reactions or behavioural changes. Incoming information is processed by the individual before a reaction is undertaken. Therefore, all alternatives of action are generally assessed in order to maximize utility. In a process called information processing, these alternatives complicate the definition of the term "utility maximization" and make it harder to simulate. It is simpler to find an action, capable of maximizing the expected reward on the one step than one capable of producing a better total reward in a long run under an uncertain environment [7]. An example of such observation is shown in Fig. 1. 


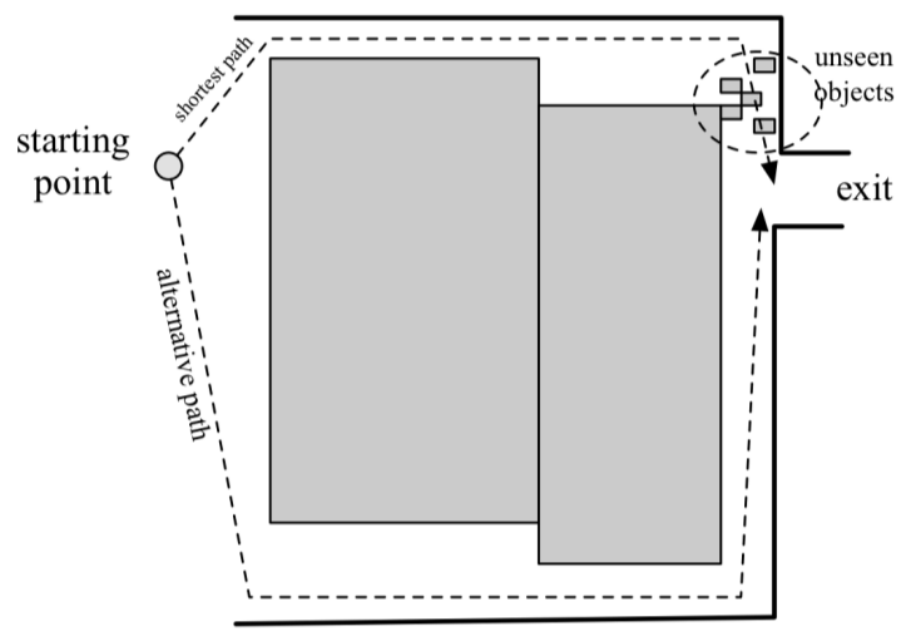

Figure 1: Best action on single step versus maximal utility in long run [8].

The result of the information processing is a decision, which together with the necessary motivation, leads to a reaction to the incoming stimulus. This sequence is also shown in Fig. 2.

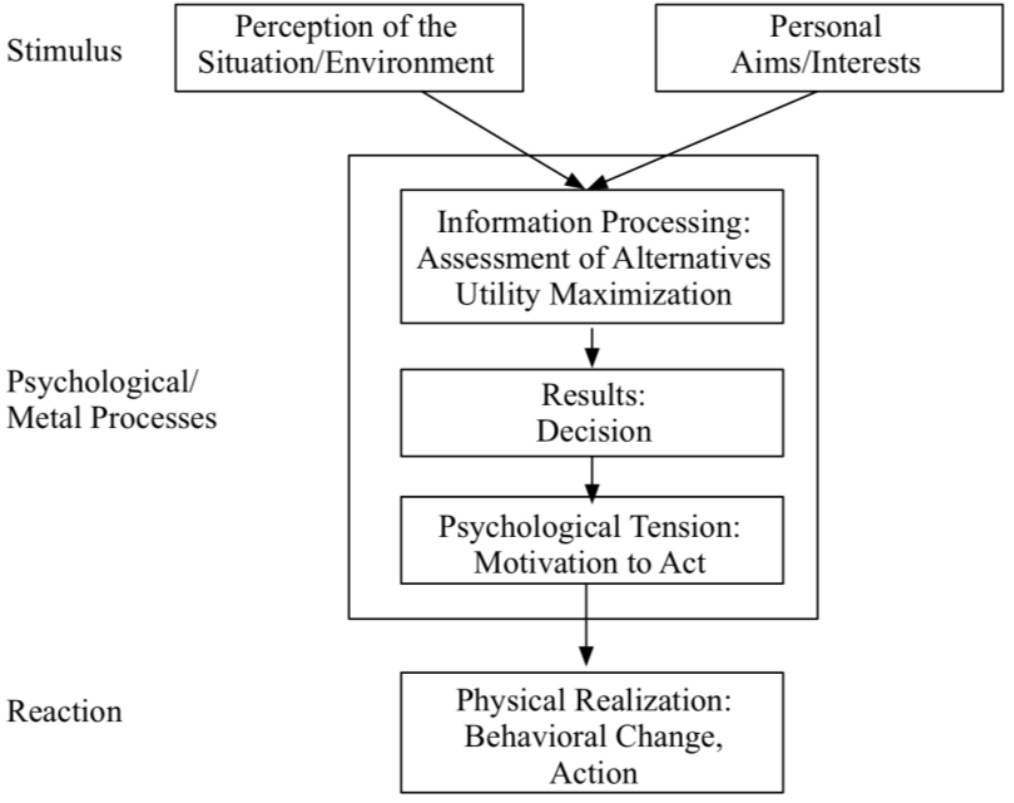

Figure 2: Schematic representation of processes leading to behavioural changes [9].

The shape of the observation ellipse is constantly changing [5], which makes its modelling even harder. Moreover, walking pedestrians only take detours or move opposite to their desired direction with strong reluctance, even if the direct way is crowded [10]. There is also evidence that people normally tend to choose the fastest route to their next target and not necessarily the shortest one [11]. Helbing et al. determined more generally that pedestrians consider both detours and their "walking comfort" to minimize the effort [12]. The routes could be approximated by polygons and are influenced by the inertia of the individual $[9,10]$.

Every pedestrian prefers an individual walking speed, which is considered as being most comfortable (i.e. referring to energy consumption) [10]. This desired speed is affected by many factors. Personal characteristics, i.e. gender, age, size or health of the pedestrian, trip characteristics, i.e. purpose, route awareness, luggage or length of the trip, infrastructure properties, i.e. type, grade, attractiveness of environment or shelter, and environmental characteristics, i.e. ambient or weather conditions [5]. In addition, the walking speed of an individual also depends on the pedestrian density [13]. However, the average walking speed is 
about $1.34 \mathrm{~m} / \mathrm{s}$. The average speed of pedestrians crossing a street is about $1.28 \mathrm{~m} / \mathrm{s}$. The speed of men is on average $10.9 \%$ higher than that of women, making an average speed of $1.41 \mathrm{~m} / \mathrm{s}$ for men and $1.27 \mathrm{~m} / \mathrm{s}$ for women. Walking speed tends to be normally distributed around the mean value with a standard deviation of $19.3 \%$ [14]. In addition, Brogan and Johnson analysed the acceleration and deceleration rates of pedestrians and found that pedestrians slow down when turning around obstacles. In general, they "begin to decelerate when 1.63 meters from the goal (...) [and] reach maximum speed at 1.82 meters from the start" [9].

The minimal spatial requirements of a pedestrian are $40 \times 40 \mathrm{~cm}$, which means 6.25 persons per square meter $[9,11,14]$. Under normal conditions, the density is lower. Weidmann reports that a density of 2 up to 2.9 persons per square meter is a normal case [14]. However, dynamic space requirements are larger than the static ones. Pedestrians need to move their legs and a security distance headways with respect to the predecessor is also required [7]. Thereby, a distance of 1 meter is postulated. The average walkway width is 0.71 $\mathrm{m}$ on even ground and $0.6 \mathrm{~m}$ on stairways [14]. However, one should note that space requirements also depend on walking speed [7].

Pedestrians also keep a minimum distance to walls and obstacles, depending on the structure of the wall and the freedom of movement. In corridors, the distance is $0.25 \mathrm{~m}$ to concrete walls and $0.2 \mathrm{~m}$ to metal walls. On sidewalks, the distance to building walls is 0.45 $\mathrm{m}$ and $0.35 \mathrm{~m}$ to fences [14]. This distance decreases as walking speed and the pedestrian density decrease [10]. Pedestrians who are resting, i.e. waiting for a train or lounging on a beach, are distributed uniformly over the available area [10]. The density is locally higher around particularly attractive places and lower with growing velocity deviation, i.e. on dance floors $[15,16]$. Groups are formed by individuals who know each another $[10,17]$. These groups behave similarly to single pedestrians, and the size of such groups is Poisson distributed [10].

In general, interactions among pedestrians can be either unilateral or bilateral. The former is described by only one pedestrian being able and required to avoid a collision and undertake action. This type of interaction is most common in unidirectional walking flows. Bilateral interactions are performed by two pedestrians meeting and subconsciously negotiating about the best manoeuvre to avoid a collision. Wolff goes further and argues that a "high degree of cooperation between pedestrians" is a fundamental part of the behaviour and condition to enable walking and crowd motion [18]. Goffman describes that pedestrians only react to and interact with others in a small circle around them. Individuals who are one or two persons away are ignored [5].

However, in situations of panic, pedestrians' behaviour changes in several ways. A "Panic stampede is one of the most tragic collective behaviours" [10] since it often causes death and injury as people are either crushed or trampled. Panic behaviour may be understandable in life-threatening situations, i.e. evacuations, fire or attacks in crowded places, but it remains a mystery in "cases of a rush for good seats at a pop concert" [10]. Despite the tragedy of such disasters, their frequency is increasing due to our growing population, easier means of transportation and larger and more frequent mass events such as concerts, demonstrations or sport events [10]. In panic situations pedestrians become nervous, which sometimes leads to blind actionist [10]. In general, individuals try to move faster than in normal situations, and interactions with others become physical in nature. This often leads to pushing. Consequently, moving becomes uncoordinated and inefficient, which often leads to congestion, especially at exits and bottlenecks [19]. The physical interactions in jammed crowds can result in pressures up to 4,500 Newton per square meter $[10,20,21]$. Moreover, pedestrians "tend to show herding behaviour" [10] like following others or doing what others do and persons who are injured or killed become "obstacles", slowing down evacuation time [10]. 


\subsection{Macroscopic level}

On a macroscopic level, self-organization and several collective patterns can be observed. Thereby, spatial-temporal patterns [10] are not planned or controlled externally i.e. by signs, conventions or rules, they emerge due to non-linear interactions among pedestrians. One of the best known and empirically observed patterns is the formation of lanes [15, 22, 23], which is shown in Fig. 3.

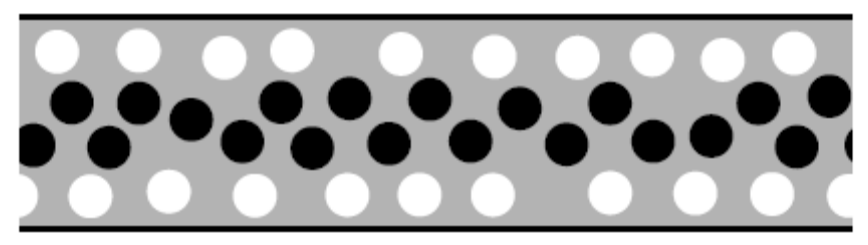

Figure 3: Formation of lanes in crowds with opposite walking direction [10].

These lanes consist of pedestrians walking in the same direction in counter flow areas. Such scenarios may appear in narrow corridors aboard ships, at pedestrian traffic lights, in shopping malls or in train stations. There are many interactions in bidirectional pedestrian flows, where each individual involved in an encounter moves to the side a bit in order to pass the others. This leads to segregation, which is quite effective as it minimizes the frequency and magnitude of sidesteps. The number of lanes depends on the width of the available space, the density and the noise level $[10,15]$. The connection between the width of the street and the number of lanes is shown in Fig. 4.

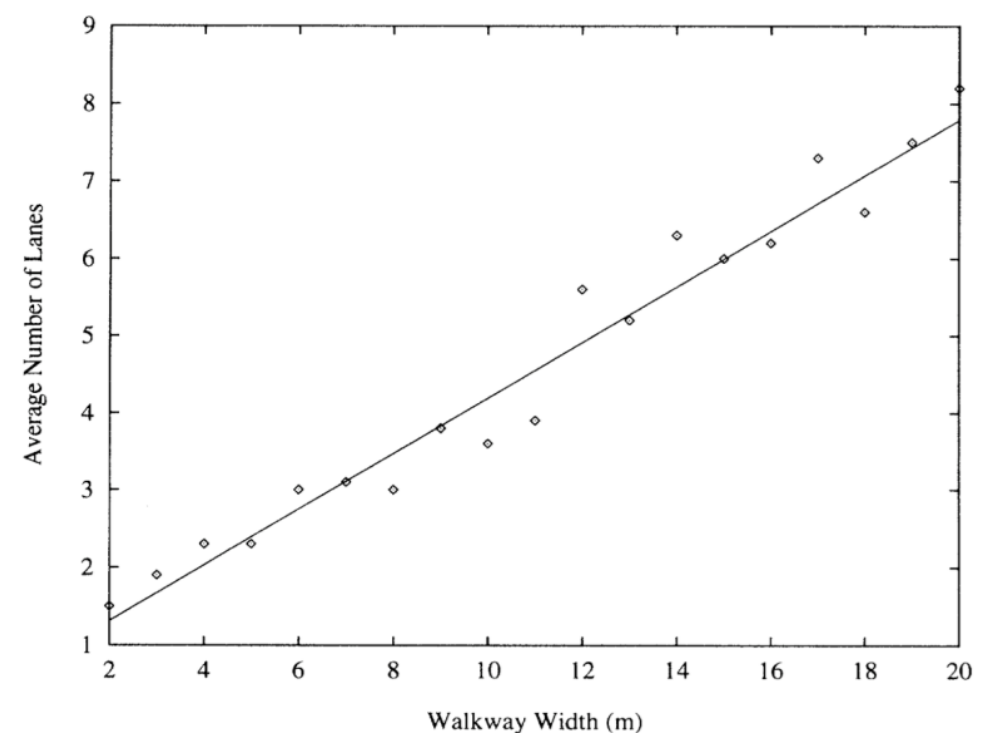

Figure 4: Average number of lanes emerging on a walkway with varying widths [13].

The stability of lanes decreases with growing rates of fluctuation $[10,13]$. To optimize the efficiency and stabilize the lanes, one could place several columns to serve a function similar to that a wall and prevent fluctuations between the lanes [10].

Another pattern occurs at bottlenecks such as doors or narrow passages. Oscillations of the walking direction at bottlenecks were observed, when people do not panic $[13,15]$. Once a pedestrian is able to pass through a narrow passage, others are easily able to follow him. Meanwhile the number and pressure at the other side of the bottleneck increases and consequently the chance of passing grows. The stream of passing pedestrians ends in a deadlock situation after which the walking direction at the narrowing changes [21]. Fig. 5 illustrates this oscillatory change. 


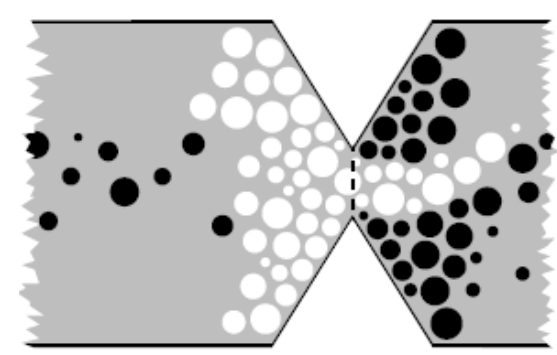

Figure 5: Oscillations of the passing direction at bottlenecks [10].

At intersections of pedestrian streams, one could observe several nondurable selforganized patterns. Unstable phases of vertical or horizontal crossing alternate with temporary roundabout traffic, as shown in Fig. 6. This roundabout traffic may cause small detours, but is more efficient on average than the alternatives of frequent deceleration, stopping and collision-avoidance.

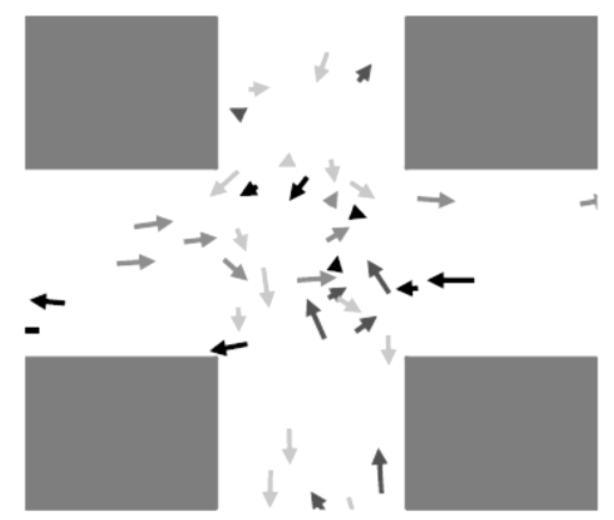

Figure 6: Self-organized, short-lived roundabout traffic in intersecting pedestrian streams [10].

In several articles, it has been conjectured that "pedestrian crowds behave similar to gases or fluids" [10]. These analogies with gas- or fluid-kinetic may especially work well in standard situations $[10,16]$. In cases of panic, the situation might change. Helbing does not assume or imply that pedestrians in panic behave relentlessly or asocial [10]. Although this may sometimes be true for pedestrians, the following characteristic features [10] are caused by other reasons. First of all, individuals become nervous, which results in a higher rate of fluctuations. Second, in order to escape from the source of panic, individuals speed up and strive for a higher velocity. Furthermore, people in complex situations often do not know what the right thing to do is. This leads to a kind of herding behaviour, where people orient themselves according the actions of their neighbours. This described behaviour, which was also mentioned in section 2.1, leads to the collective effect in panic situations.

Due to the higher rates of fluctuations caused by the pedestrians' nervousness and the crowd density in general, the lanes in bidirectional flows are destroyed. But instead of a "disordered gaseous state" [10], a solid and blocked state is formed [22]. Helbing et al. [10] call this paradoxical transition "freezing by heating". Fig. 7 illustrates this effect in a corridor.

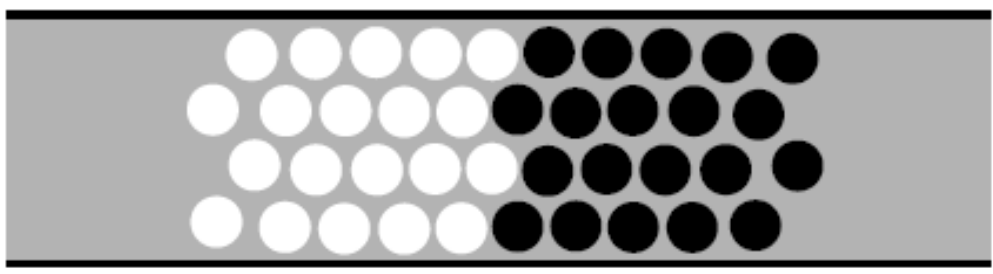

Figure 7: Formation of a blocked, "frozen" state in panic situations [10]. 
This blocked state is not what one would expect as an equilibrium, but is rather a metastable state with structural perturbations [10].

At exits, the outflow of pedestrians is coordinated and regular if the desired velocities are normal. In panic and rush situations with desired speeds of $1.5 \mathrm{~m} / \mathrm{s}$ and more, one could observe arch-like blocking of the exit and clogging, as shown in Fig. 8.

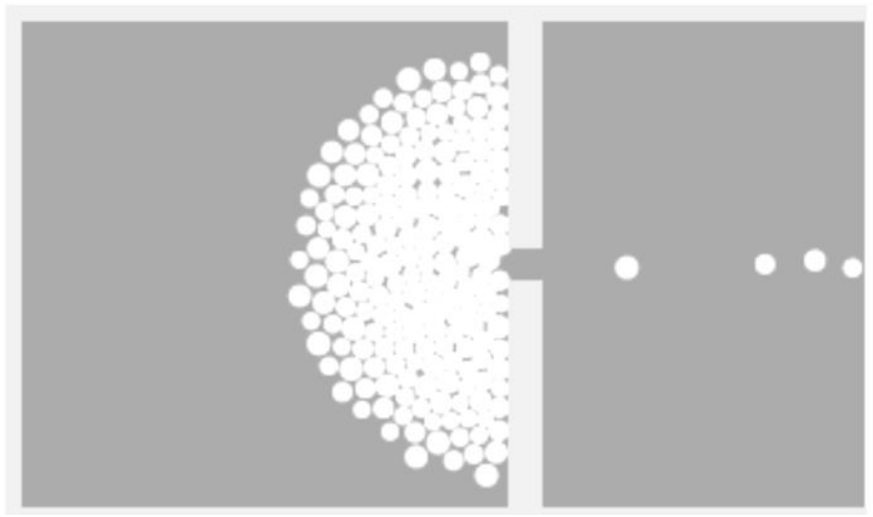

Figure 8: Arch-like clogging at an exit [10].

When the arches break due to the increasing pressure of pedestrians, individuals leave in "avalanche-like bunches" [10,22]. With increasing desired velocities, time to escape the room is increasing as well. Moreover, individuals get injured due to the rising pressure which increases the escape time again. Fig. 9 illustrates this relation. Injuries happen if the "sum of the magnitudes of the radial forces acting on them (the pedestrians) divided by their circumference exceeds a pressure of $1600 \mathrm{Nm}$ " [10, 21]. Finally, pedestrians increase the average time to pass the bottleneck if they increase their desired velocities and start rushing and pushing. This effect, which is referred to as "faster-is-slower-effect" [10], is especially tragic in life-threatening situations.
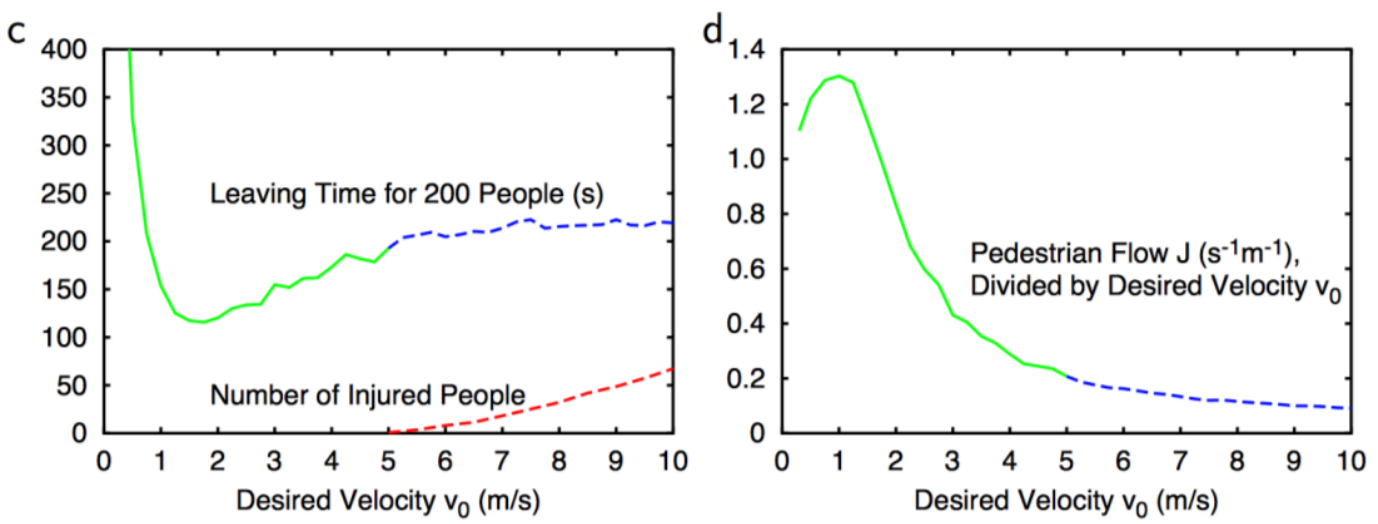

Figure 9: Analysis of evacuation scenarios [10].

The time needed for 200 people to leave a room decreases under normal conditions with increasing desired velocities. Desired speeds above $1.5 \mathrm{~m} / \mathrm{s}$ reduce the efficiency of leaving. At desired velocities of $5 \mathrm{~m} / \mathrm{s}$ more people are injured [10]. This phenomenon could be avoided by constructing public places with a minimum number of bottlenecks. However, such jamming may also occur at widenings, as is shown in Fig. 10. In the given case, the desired velocities are $2 \mathrm{~m} / \mathrm{s}$, the corridor is $3 \mathrm{~m}$ wide and $15 \mathrm{~m}$ long, in the middle it is $9 \mathrm{~m}$ wide. 


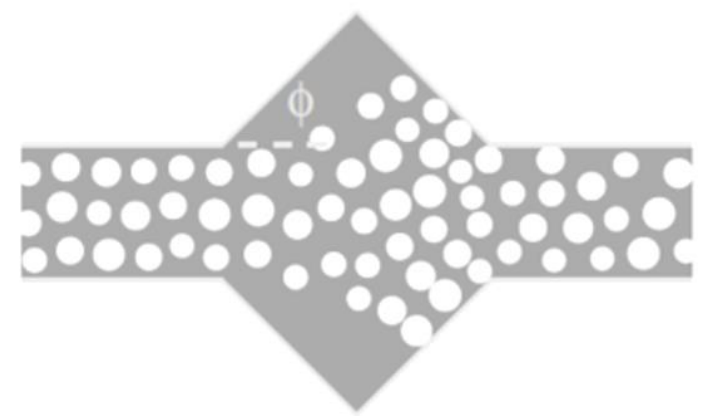

Figure 10: Jamming in a corridor with a wider area [10].

Pedestrians with a higher desired velocity try to overtake others in the wider area. The end of that area is similar to a bottleneck and pedestrians try to squeeze again in the mainstream which leads to jams. This effect occurs when the corridor is narrow, the density of pedestrians is high and desired velocities are different or high in general [10].

Panic with all the described results could also occur without any obvious reason $[10,15]$. This so-called "phantom panic" occurs due to the "faster-is-slower" effect described above. If a crowd intends to leave, a small counter flow of pedestrians could cause delays [20]. Stopped pedestrians in the back of the crowd cannot see the reason for the slowdown, become impatient and start pushing. To prevent this effect, one should have wide enough exits and no counter flows if big crowds want to leave [24].

\section{ENVIRONMENT SPACE}

Pedestrian behaviour has often been modelled for several reasons. Simulations can help to understand crowd behaviour better or to analyse macroscopic and collective patterns. Furthermore, one could i.e. simulate evacuation scenarios in advance to increase quality in the planning of public buildings and plazas. Therefore, some existing approaches are described in this section.

To achieve complex behaviour, stochastic description is often chosen instead of a deterministic one [25]. In complex situations, even a minor change in the environment or minor events could lead to very different behaviour which would be very difficult to describe using a deterministic approach. The stochastic approach considers that one does not usually have full knowledge about the system and its dynamics. Furthermore, average processes are described and questions about the probability of a certain event can be answered [25]. It is not necessary for a reliable simulation to know how a certain pedestrian acts, i.e. whether he/she will turn left at the next opportunity or not. It is sufficient to know what percentage of pedestrians turn left.

Most models use a microscopic approach which models the individual behaviour of a pedestrian. All macroscopic behaviour is a result of the interactions among the pedestrians. This approach could usually be divided into space-discrete and space-continuous. The main difference is about the motion and the physical dimensions of a pedestrian [26]. In spacecontinuous models a person could have any bodily dimension and move freely in the available space. In space-discrete is discretized and pedestrians move on a lattice. Consequently, the shape and dimension is limited by the cell-size and the motion occurs from cell to cell, similar to chess. Discrete models are perfectly suited for high-performance computing and mathematical analysis. On the other hand, continuous models are based on coupled differential equations, which can often only be solved numerically and are therefore harder to analyse mathematically [25]. In addition, space-discrete as space-continuous models are discrete in time. 


\subsection{Space-discrete models}

Space-discrete models are very close to the "general strategy of modelling (elementary) forces on a microscopic level" [25]. One example are active walker models [12]. Here the pedestrian modifies the path he or she is taking and leaves a trace. These models could be used to describe trail formation, for example. Discrete models for pedestrian dynamics have been developed in [1]. Most work has focused on the occurrence of selected collective effects of pedestrian behaviour, e.g. jamming with growing density. Schadschneider developed a type of cellular automaton which can reproduce all collective patterns [25]. He uses an idea similar to chemotaxis to implement the interactions among pedestrians. Every individual leaves a virtual trace which influences the motion and action of other pedestrians. He generalizes this idea to a "so-called floor field" which includes the virtual trace and a static component. The latter does not change with time and is used to model walls, preferred areas or obstacles. Pedestrians react to both kinds of floor fields. For more information and a detailed model description see reference [25]. Kretz took the same approach and published the "Floor fieldand agent-based simulation tool model". He also reviewed some other discrete pedestrian models [13].

\subsection{Space-continuous models}

Space-continuous models are very realistic in representing physical dimensions and motion. Many different simulation models have been developed in the past decades, i.e., queueing models [27] or route choice models [28]. According to the above-mentioned approach that pedestrians behave similar to gases or fluids, Henderson proposed a model as well [10]. One continuous model developed by Helbing et al. is very well known and will be described in the following. They proposed a so-called social force model $[9,10]$, which suggests that pedestrians are subject to social forces. These forces are not directly exerted by the environment to the pedestrian's body. "It is rather the quantity that describes the concrete motivation to act" [9] and the direction and amount of behavioural changes. These social forces are put into mathematical terms requiring vector additivity of the forces that represent different environmental influences [9, 19]. The central equation of motion describes the change of the location $x_{i}(t)$ of pedestrian $i$ at time $t[10]$ :

$$
\frac{d x_{i}(t)}{d t}=v_{i}(t)
$$

Furthermore, if $f_{i}(t)$ denotes the sum of all forces influencing pedestrian $i, m_{i}$ is the mass and $\xi_{i}(t)$ are individual fluctuations, the velocity $v_{i}(t)$ changes are given by the term of acceleration [10].

$$
m_{i} \frac{d v_{i}}{d t}=f_{i}(t)+\xi_{i}(t)
$$

Moreover, Helbing formulates equations for acceleration, deceleration, a repulsive effect to other pedestrians, an attractive effect towards window displays or special attractions and an equation for the joining behaviour of friends, families or tourist groups. All these equations can be seen in references $[9,10,19]$. The model was used and further developed according to different approaches, for example in combination with a genetic fuzzy system in order to research pedestrian's steering behaviour under normal circumstances through a built environment. Thereby, the social force model is used to quantify the environmental stimuli [29].

Furthermore, Gipps and Marksjö proposed a discrete model called the "micro simulation model for pedestrian flows" [30] that analyses individual behaviour of entities, while Qiu and $\mathrm{Hu}$ concentrate on group structures in pedestrian crowds [31]. The authors claim, that a cluster of pedestrians can lead to slower movement and its consideration in models would 
therefore improve results, especially in constrained areas [31]. The pedestrian behaviour on a micro view for pedestrian flow through unusual space was studied by Guo and Tang [32]. The authors analysed pedestrian behaviour on walkways with corners, where the crowd forms different shapes. The paper concludes that the shape of a corner influences the crowd behaviour strongly, for example each increasing turning angle of a corner makes the pedestrian queue become more and more unordered [32].

\section{BEHAVIOUR TYPE}

Classical models have shown good results in modelling pedestrian behaviour but some limitations of these models remain [33]. Most environments have dynamic properties and some unpredictable situations could occur potentially. Those cases make it difficult to model and simulate pedestrian behaviour realistically [34]. Assuming a same set of rules for every case, it is not possible for simulated pedestrians to learn from each other and from the environment, like a human would [33]. To overcome these limitations, researchers and scientists have tried to develop new models based on learning techniques, called learningbased models.

In this section, some existing models will be described and discussed in two categories: classical (pre-programed) and modern (learning-based) models. Like previously mentioned, they use the microscopic approach and are discrete in time.

\subsection{Pre-programed behaviour}

Teknomo et al. [35] divided the classical microscopic pedestrian simulation models into three different categories: benefit cost cellular, magnetic force models and social force models. On the other hand Zheng et al. [4] discussed existing models for crowd evacuation based on seven methodological approaches: cellular automata models, lattice gas models, social force models, agent-based models, fluid dynamic models, game theoretic models and approaches based on experiments with animals, where the last three approaches have not gained much attention with low number of publications and citations. For comparison, Table I shows some well-known and frequently cited models in three categories based on pedestrian interactions properties. They are force-, grid- and agent-based models [33]. Cellular automata, lattice gas models belong to grid-based models.

Table I: Microscopic rule-based models [35].

\begin{tabular}{|c|l|l|l|}
\hline Approach & \multicolumn{1}{|c|}{ Source } & \multicolumn{1}{|c|}{ Space } & \multicolumn{1}{c|}{ Typical Behaviour } \\
\hline \multirow{4}{*}{ Grid-based } & Burstedde et al. [36] & Discrete & Evacuation, lane formation \\
\cline { 2 - 5 } & Blue and Adler [37] & Discrete & Bi-directional movement \\
\cline { 2 - 5 } & Höcker et al. [38] & Discrete & Path finding \\
\cline { 2 - 5 } & $\begin{array}{l}\text { Kirchner and } \\
\text { Schadschneider [39] }\end{array}$ & Discrete & Evacuation, Arch-like clogging \\
\hline \multirow{5}{*}{ Force-based } & Rogsch [26] & Continuous & Clogging, faster-is-slower, mass behaviour \\
\cline { 2 - 4 } & Chraibi et al. [40] & Continuous & Oscillations, overlapping \\
\cline { 2 - 4 } & Helbing and Molnar [16] & Continuous & Lane formation, oscillatory change \\
\cline { 2 - 4 } & Mehran et al. [41] & Discrete & Abnormal behaviour \\
\hline \multirow{3}{*}{ Agent-based } & Pelechano et al. [42] & Continuous & Queuing, pushing \\
\cline { 2 - 4 } & Toyama et al. [43] & Discrete & Lane formation, herding, obstacle avoidance \\
\cline { 2 - 4 } & Brau et al. [44] & Continuous & Decision, escape, risk \\
\hline
\end{tabular}




\subsection{Learning-based behaviour}

To tackle the difficult relationship between individual behaviour and group behaviour in microscopic models, Martinez-Gil et al. [34] designed a Multi-Agent ReinforcementLearning-based pedestrian simulation framework called MARL-Ped. Thereby, each agent learns to navigate autonomously in the environment based on a model-free reinforcement learning algorithm. The authors proposed two learning algorithms based on vector quantization and Q-learning, one of the most popular reinforcement learning techniques [7]. The first one, IN-VQQ has a fixed number of agents while the other one, IT-VQQ increases the number of agents after each iteration. Vector quantization was used to generate the states of the model. Q learning, was used in the learning process of the agents, which was the key to get realistic agent's behaviour [34]. The model and its results are comparable to the original work of Helbing [7, 12]. Recently, Ma et al. [33] developed a novel approach based on artificial neural network with a multi-layered preceptor (ANN-MLP) which allows them to build a non-linear, adaptive and open model. In this article, they applied a semiautomatic approach developed by Teknomo [45] to extract pedestrian data from a video and used it for the training and testing process. This seems an interesting approach to gain data for learningbased models since the pedestrian data is almost classified and therefore almost unavailable. Not long ago, Barlas et al. likewise introduced a very useful tool called KE. This tool aims at achieving the automation of generating input data for simulation models based on exiting data sources. It follows the Core Manufacturing Simulation Data standard, which is widely cited in the simulation literature [46].

There are not so much learning-based models as pre-programed models. Furthermore, these models have also got less attention from researcher and scientists according to the number of publications and citations. This could be explained by how recent the learning techniques researched are. There is still limited application possibility for simulation models. For clarification proposes, some well-known models are shown in Table II.

Table II: Microscopic learning-based models.

\begin{tabular}{|l|l|l|l|l|}
\hline \multicolumn{1}{|c|}{ Source } & \multicolumn{1}{c|}{ Space } & \multicolumn{1}{c|}{ Category } & Learning techniques & \multicolumn{1}{c|}{ Typical Behaviour } \\
\hline Ma et al. [33] & Continuous & Agent-based & Neural network & Both-directional movement \\
\hline $\begin{array}{l}\text { Martinez-Gil et } \\
\text { al. [34] }\end{array}$ & Continuous & Agent-based & $\begin{array}{l}\text { Reinforcement } \\
\text { learning }\end{array}$ & Navigation \\
\hline $\begin{array}{l}\text { Zainuddin and } \\
\text { Aik [47] }\end{array}$ & Discrete & $\begin{array}{l}\text { Grid-based, } \\
\text { Agent-based }\end{array}$ & $\begin{array}{l}\text { Probabilistic neural } \\
\text { network }\end{array}$ & Evacuation \\
\hline Oliver et al. [48] & Discrete & Agent-based & Machine learning & $\begin{array}{l}\text { Walk together, follow, reach } \\
\text { target, change direction }\end{array}$ \\
\hline
\end{tabular}

\section{CONCLUSION AND OUTLOOK}

In the paper at hand, an overview of existing knowledge about modelling the behaviour of pedestrians in public areas is given. How do pedestrians move and how is the interaction with others? What is the average speed and how much space does an individual require in different situations? What happens in situations of panic? All these questions are answered in section 2. Moreover, in section 2 various collective phenomena such as lane formation in bidirectional flows, oscillation at bottlenecks and self-organization effects at intersections are shown. In panic situations like an evacuation, collective patterns change and need to be modelled differently. Lanes break down to a blocked and "frozen" state, arch-like clogging at bottlenecks were observed and jamming in corridors with widenings is not unusual. In general, the faster-is-slower effect occurs in situations of panic, and huge pressure is built up 
by pushing pedestrians, often leading to injury or even death. Not considering these effects in simulations would lead to wrong results and dangerous implications.

In section 3 and section 4, some modelling approaches are presented according to their environment space and behaviour type. Some of them focused on specific effects of human behaviour; others tried to be as realistic as possible. Each approach was chosen for answering specific research questions, which needs to be considered in the evaluation. Nevertheless, a good and realistic model should be able to reproduce all the above-mentioned collective patterns. Learning-based modelling approaches might be used to build more realistic models but there is a serious lack of data for the learning techniques available. A model should also not only depend on artificial data and assumptions because it might lightly represent the subjective opinion of the model builder. Many approaches have been developed to overcome this problem. Collecting and analysing pedestrian data using camera and sensor seems the most useful one. For example, Helbing et al. [49] have analysed a video recording disaster of more than two million pedestrians during the Hajj to gain the data for his model, which became one of the most popular crowd disaster models. Unfortunately, the price for such solution is still very high, as reported by Miovision - one of the most trusted data platforms [50]. However, modelling and simulation is an excellent approach in planning and optimizing pedestrian areas. It could help to make public areas, i.e. buildings, offices, train stations, ships, etc., more secure by reducing evacuation time and optimizing evacuation routes. Moreover, a simulation can cover different scenarios and is less dangerous and less expensive than real experiments.

For future research, we suggest the collection of more detailed data and information on behaviour in panic situations. This could help to make models even more realistic and feasible. For example, more complex interactions between pedestrians or acoustic information exchange could be adopted. Also, smoke, toxicities and other things that affect pedestrians' perception, mental strength and consciousness could be analysed and adapted to models.

\section{REFERENCES}

[1] Klüpfel, H.; Meyer-König, T.; Wahle, J.; Schreckenberg, M. (2001). Microscopic simulation of evacuation processes on passenger ships, Bandini, S.; Worsch, T. (Eds.). Theory and practical issues on cellular automata, Springer, London, 63-71, doi:10.1007/978-1-4471-0709-5_8

[2] Ahmed, R.; Shah, M.; Umar, M. (2016). Concepts of simulation model size and complexity, International Journal of Simulation Modelling, Vol. 15, No. 2, 213-222, doi:10.2507/ ijsimm15(2)2.317

[3] Barlas, P.; Heavey, C.; Dagkakis, G. (2015). An open source tool for automated input data in simulation, International Journal of Simulation Modelling, Vol. 14, No. 4, 596-608, doi: $\underline{10.2507 / I J S I M M 14(4) 3.306}$

[4] Zheng, X.; Zhong, T.; Liu, M. (2009). Modeling crowd evacuation of a building based on seven methodological approaches, Building and Environment, Vol. 44, No. 3, 437-445, doi:10.1016/j.buildenv.2008.04.002

[5] Goffman, E. (1971). Relations in public: Microstudies of the public order, Basic Books, New York

[6] Hoogendoorn, S. P.; Daamen, W. (2005). Pedestrian behavior at bottlenecks, Transportation Science, Vol. 39, No. 2, 147-159, doi:10.1287/trsc.1040.0102

[7] Sutton, R. S.; Barto, A. G. (1998). Reinforcement learning: An introduction, MIT Press, Cambridge

[8] Kneidl, A.; Hartmann, D.; Borrmann, A. (2014). Using a multi-scale model for simulating pedestrian behavior, Weidmann, U.; Kirsch, U.; Schreckenberg, M. (Eds.). Pedestrian and Evacuation Dynamics 2012, Springer, 1029-1038, doi:10.1007/978-3-319-02447-9_85

[9] Brogan, D. C.; Johnson, N. L. (2003). Realistic human walking paths, $16^{\text {th }}$ International Conference on Computer Animation and Social Agents, 94-101 
[10] Helbing, D.; Farkas, I. J.; Molnar, P.; Vicsek, T. (2002). Simulation of pedestrian crowds in normal and evacuation situations, Schreckenberg, M.; Sharma, S. D. (Eds.). Pedestrian and evacuation dynamics, Springer, Berlin, 21-58

[11] Ganem, J. (1998). A behavioral demonstration of Fermat's principle, The Physics Teacher, Vol. 36, No. 2, 76-78, doi:10.1119/1.880019

[12] Helbing, D.; Keltsch, J.; Molnar, P. (1998). Modelling the evolution of human trail systems, Nature, Vol. 388, No. 6637, 47-50, doi:10.1038/40353

[13] Kretz, T. (2007). Pedestrian traffic - simulation and experiments, PhD Thesis, Department of Physics, University Duisburg-Essen, Duisburg

[14] Weidmann, U. (1992). Transporttechnik der fussgänger, Institut für Verkehrsplanung, Transporttechnik, Strassen- und Eisenbahnbau, Schriftenreihe des IVT Nr. 90, ETH Zürich, Zürich

[15] Helbing, D. (2013). Verkehrsdynamik: neue physikalische Modellierungskonzepte, SpringerVerlag, Berlin

[16] Helbing, D.; Molnar, P. (1995). Social force model for pedestrian dynamics, Physical Review E, Vol. 51, No. 5, 4282-4286, doi:10.1103/physreve.51.4282

[17] Jayalath, D. D. A. C. J.; Wimalaratne, S. P. W.; Karunananda, A. S. (2016). Modelling goal selection of characters in primary groups in crowd simulations, International Journal of Simulation Modelling, Vol. 15, No. 4, 585-596, doi:10.2507/ijsimm15(4)1.323

[18] Daamen, W.; Hoogendoorn, S. (2003). Experimental research of pedestrian walking behavior, Transportation Research Record: Journal of the Transportation Research Board, Vol. 1828, 2030, doi:10.3141/1828-03

[19] Helbing, D. (1993). Stochastische Methoden, nichtlineare Dynamik und quantitative Modelle sozialer Prozesse, Shaker, Aachen

[20] Elliott, D.; Smith, D. (1993). Football stadia disasters in the United Kingdom: learning from tragedy?, Industrial \& Environmental Crisis Quarterly, Vol. 7, No. 3, 205-229, doi:10.1177/ 108602669300700304

[21] Smith, R. A.; Dickie, J. F. (1993). Engineering for crowd safety: Proceedings of the International Conference on Engineering for Crowd Safety, Elsevier, London

[22] Helbing, D. (2001). Die wundervolle Welt aktiver Vielteilchensysteme: Autos, Fußgänger, Vögel oder andere „motorisierte” Teilchen lassen sich durch relativ einfache Verallgemeinerungen der Newtonschen Gleichungen beschreiben, Physikalische Blätter, Vol. 57, No. 1, 27-33, doi: $10.1002 / \mathrm{phbl} .20010570110$

[23] Helbing, D.; Molnar, P. (1998). Self-organization phenomena in pedestrian crowds, Schwitzer, F. (Ed.). Self-organization of Complex Structures: From Individual to Collective Dynamics, Gordon and Breach Science Publishers, Amsterdam, 569-577

[24] Helbing, D.; Farkas, I.; Vicsek, T. (2000). Simulating dynamical features of escape panic, Nature, Vol. 407, No. 6803, 487-490, doi:10.1038/35035023

[25] Schadschneider, A. (2001). Cellular automaton approach to pedestrian dynamics - Theory, Schreckenberg, M.; Sharma, S. D. (Eds.). Pedestrian and Evacuation Dynamics, Springer, Berlin, 75-86

[26] Rogsch, C. (2005). Vergleichende Untersuchungen zur dynamischen Simulation von Personenströmen, Diplomarbeit, Bergische Universität Wuppertal, Wuppertal

[27] Roy, J. R. (1992). Queuing in spatially dispersed public facilities, IV World Congress of the Regional Science Association International, 17-25

[28] Vogt, R.; Nikolaidis, I.; Gburzynski, P. (2012). A realistic outdoor urban pedestrian mobility model, Simulation Modelling Practice and Theory, Vol. 26, 113-134, doi:10.1016/j.simpat. 2012.04 .006

[29] Nasir, M.; Lim, C. P.; Nahavandi, S.; Creighton, D. (2014). A genetic fuzzy system to model pedestrian walking path in a built environment, Simulation Modelling Practice and Theory, Vol. 45, 18-34, doi:10.1016/j.simpat.2014.03.002

[30] Gipps, P. G.; Marksjö, B. (1985). A micro-simulation model for pedestrian flows, Mathematics and Computers in Simulation, Vol. 27, No. 2-3, 95-105, doi:10.1016/0378-4754(85)90027-8

[31] Qiu, F.; Hu, X. (2010). Modeling group structures in pedestrian crowd simulation, Simulation Modelling Practice and Theory, Vol. 18, No. 2, 190-205, doi:10.1016/j.simpat.2009.10.005 
[32] Guo, R.-Y.; Tang, T.-Q. (2012). A simulation model for pedestrian flow through walkways with corners, Simulation Modelling Practice and Theory, Vol. 21, No. 1, 103-113, doi:10.1016/ j.simpat.2011.10.007

[33] Ma, Y.; Lee, E. W. M.; Yuen, R. K. K. (2016). An artificial intelligence-based approach for simulating pedestrian movement, IEEE Transactions on Intelligent Transportation Systems, Vol. 17, No. 11, 3159-3170, doi:10.1109/tits.2016.2542843

[34] Martinez-Gil, F.; Lozano, M.; Fernández, F. (2015). Strategies for simulating pedestrian navigation with multiple reinforcement learning agents, Autonomous Agents and Multi-Agent Systems, Vol. 29, No. 1, 98-130, doi:10.1007/s10458-014-9252-6

[35] Teknomo, K.; Takeyama, Y.; Inamura, H. (2000). Review on microscopic pedestrian simulation model, Proceedings of the Japan Society of Civil Engineering Conference, 536-537

[36] Burstedde, C.; Klauck, K.; Schadschneider, A.; Zittartz, J. (2001). Simulation of pedestrian dynamics using a two-dimensional cellular automaton, Physica A: Statistical Mechanics and its Applications, Vol. 295, No. 3-4, 507-525, doi:10.1016/s0378-4371(01)00141-8

[37] Blue, V. J.; Adler, J. L. (2001). Cellular automata microsimulation for modeling bi-directional pedestrian walkways, Transportation Research Part B: Methodological, Vol. 35, No. 3, 293-312, doi: 10.1016/s0191-2615(99)00052-1

[38] Höcker, M.; Berkhahn, V.; Kneidl, A.; Borrmann, A.; Klein, W. (2010). Graph-based approaches for simulating pedestrian dynamics in building models, Menzel, K.; Scherer, R. (Eds.). eWork and eBusiness in Architecture, Engineering and Construction, CRC Press, Taylor \& Francis Group, London, 389-394

[39] Kirchner, A.; Schadschneider, A. (2002). Simulation of evacuation processes using a bionicsinspired cellular automaton model for pedestrian dynamics, Physica A: Statistical Mechanics and its Applications, Vol. 312, No. 1-2, 260-276, doi:10.1016/s0378-4371(02)00857-9

[40] Chraibi, M.; Seyfried, A.; Schadschneider, A. (2010). Generalized centrifugal-force model for pedestrian dynamics, Physical Review E, Vol. 82, No. 4, Paper 046111, doi:10.1103/ physreve.82.046111

[41] Mehran, R.; Oyama, A.; Shah, M. (2009). Abnormal crowd behavior detection using social force model, IEEE Conference on Computer Vision and Pattern Recognition, 935-942

[42] Pelechano, N.; Allbeck, J. M.; Badler, N. I. (2007). Controlling individual agents in high-density crowd simulation, Proceedings of the 2007 ACM SIGGRAPH/Eurographics Symposium on Computer Animation, 99-108

[43] Toyama, M. C.; Bazzan, A. L. C.; Da Silva, R. (2006). An agent-based simulation of pedestrian dynamics: from lane formation to auditorium evacuation, Proceedings of the $5^{\text {th }}$ International Joint Conference on Autonomous Agents and Multiagent Systems, 108-110

[44] Braun, A.; Bodmann, B. E. J.; Musse, S. R. (2005). Simulating virtual crowds in emergency situations, Proceedings of the ACM Symposium on Virtual Reality Software and Technology, 244-252

[45] Teknomo, K. (2002). Microscopic pedestrian flow characteristics: Development of an image processing data collection and simulation model, $\mathrm{Ph} . \mathrm{D}$. Thesis, Tohoku University, Sendai

[46] Ahmed, R.; Shah, M. (2015). Exploring the context and practices of expert simulation modellers, International Journal of Simulation Modelling, Vol. 14, No. 2, 265-277, doi:10.2507/ IJSIMM14(2)7.297

[47] Zainuddin, Z.; Aik, L. E. (2012). Intelligent exit-selection behaviors during a room evacuation, Chinese Physics Letters, Vol. 29, No. 1, Paper 018901, doi:10.1088/0256-307x/29/1/018901

[48] Oliver, N. M.; Rosario, B.; Pentland, A. P. (2000). A Bayesian computer vision system for modeling human interactions, IEEE Transactions on Pattern Analysis and Machine Intelligence, Vol. 22, No. 8, 831-843, doi:10.1109/34.868684

[49] Helbing, D.; Johansson, A.; Al-Abideen, H. Z. (2007). Dynamics of crowd disasters: An empirical study, Physical Review E, Vol. 75, No. 4, Paper 046109, doi:10.1103/ physreve.75.046109

[50] Miovision. Collecting and Analyzing Data, from: https://miovision.com/walkable-bikeable-cityguide/pedestrian-counts-data/, accessed on 13-03-2017 